\title{
INCREMENTO DIAMÉTRICO DE Guarea macrophylla VAHL. Y Calycophyllum spruceanum (BENTHAM) HOOKER F. EX. SCHUMANN EN BOSQUES INUNDABLES DE LA AMAZONÍA PERUANA
}

\author{
Juan Baluarte ${ }^{1}$, Gustav Nebel ${ }^{2}$
}

\section{RESUMEN}

Esta nota técnica presenta algunos resultados sobre el incremento diamétrico de dos especies forestales, con madera de valor comercial, que medran en los bosques inundables de la Amazonía peruana. Con esta investigación se busca resultados que puedan ser útiles en la planificación del manejo sostenible de las especies. Con base a datos de incremento diamétrico tomados en parcelas permanentes de muestreo, se presenta resultados del análisis por clases diamétricas y simples modelos de incremento de diámetro; sin embargo, se necesita incluir factores de competencia en los análisis para poder comprender por que unos individuos crecen más que otros. La baja densidad de algunas especies en bosques tropicales hace difícil tener una base de datos suficiente para un análisis adecuado, como es el caso de Calycophyllum spruceanum, cuya presencia de individuos jóvenes fue escasa en las parcelas de crecimiento, estudiadas.

Palabras clave: Incremento diamétrico Guarea macrophylla, Calycophyllum spruceanum, Amazonía peruana.

\begin{abstract}
This technical note presents results on the diameter growth of two forest tree species producing commercially valuable wood, which grow in the flood plain forests of the Peruvian Amazon. The purpose is to provide results that can be used in the planning of a sustainable management of the species. Based on diameter increment data from permanent sample plots, results are presented on the diameter growth in diameter

1 Instituto de Investigaciones de la Amazonía Peruana (IIAP), Av. Abelardo Quiñonez. km 2.5, Iquitos, Perú. E-mail: jbaluarte@iiap.org.pe

2 Universidad Real de Veterinaria y de Agricultura (KLV), Rolighedsvej 23, DK DK-1958 Frederiksberg C, Dinamarca. E-mail: gne@klv.dk
\end{abstract}


classes and according to simple growth models. However, it is necessary to include competition factors in the analyses to be able to understand why some individuals grow better than others. The low density of some species in the tropical forests makes it difficult to get a database allowing an adequate analysis, which is the case for Calycophyllum spruceanum where only few young individuals were present in the permanent plots included in the study.

Key Words: Diameter increment, Guarea macrophylla, Calycophyllum spruceanum, Peruvian Amazon.

\section{INTRODUCCIÓN}

Muchas de las especies forestales de los bosques inundables de la Amazonía peruana son utilizadas para madera y productos forestales no maderables. Estos bosques tienen un rol productivo importante debido a factores como acceso relativamente fácil y bajos costos de extracción. Para poder manejar las poblaciones de las especies extraídas se necesita, entre otras condiciones, contar con información sobre su ecología y crecimiento. En esta nota técnica se enfatiza en el crecimiento, brindando información sobre el incremento diamétrico de dos especies forestales maderables de valor comercial.

Guarea macrophylla Vahl, de la familia Meliaceae, es conocida localmente como "requia colorada". Es una especie relativamente abundante en los bosques inundables, cuenta con una madera de color rojizo, densidad intermedia, es utilizada en ebanistería para muebles y molduras. Calicophyllum spruceanum (Bentham) Hooker f. ex Schumann de la familia Rubiaceae, cuyo nombre común es "capirona", tiene características ecológicas de pionera; la madera tiene densidad muy alta, sumamente dura y es utilizada localmente como leña y madera redonda para construcciones rurales; en Ucayali, últimamente ha sido explotada en grandes volúmenes, para su uso en pisos y carpintería. 


\section{2. ÁREA DE ESTUDIO}

Las parcelas permanentes fueron establecidas en bosques inundables de las zonas de estudio de Braga-Supay, Lobillo e Iricahua ubicadas en los alrededores de Jenaro Herrera ( $4^{\circ} 55^{\prime}$ 'sur, $73^{\circ} 44^{\prime}$ ' oeste), río Ucayali, Amazonía peruana. Las descripciones generales de los bosques inundables y de las zonas de estudio fueron reportados por Nebel (1999).

\section{DISTRIBUCIÓN, MORFOLOGÍA Y PROPIEDADES DE LA MADERA}

G. macrophylla es una especie dioica, perteneciente a la familia Meliaceae. El árbol es recto, alcanza fácilmente $20 \mathrm{~m}$ o más de altura y $70 \mathrm{~cm}$ de diámetro; la corteza externa es rosada con ritidoma que se desprende en placas; hojas pinnadas, 2-5 pares de folíolos, opuestos y glabros (Spichiger et al. 1990).

Esta especie se distribuye entre 0-500 msnm en la Amazonía (Colombia, Ecuador, Perú y Bolivia) hasta el río Acre (Brasil). Colectado con frecuencia en las riberas de los ríos (Spichiger et al., 1990; Brako \& Zarucchi, 1993).

G. macrophylla tiene una madera de color rojizo con una densidad de $0.60 \mathrm{~g} / \mathrm{cm}^{3} \mathrm{y}$ una buena durabilidad natural, cuenta con grano recto, textura media, fácil aserrío y trabajabilidad, siendo utilizado en ebanistería para muebles y molduras (JUNAC, 1981; Aróstegui, 1982; Toledo, 1994).

C. spruceanum es una especie de la familia Rubiaceae. El árbol es recto con copa heterogénea, hasta $30 \mathrm{~m}$ de altura, fácilmente reconocible por su tronco liso y brillante, rojizo, verduzco o grisáceo, con ritidoma coriáceo, caduco anualmente; hojas simples, opuestas y pecioladas; flores pequeñas, blancas y aromáticas (Lao, 1986).

Esta especie se distribuye en toda la región tropical de Latinoamérica, entre 0-1000 msnm, desde Bolivia hasta la Amazonía brasileña y peruana, particularmente habita fértiles áreas forestales recientemente intervenidas y ocasionalmente inundadas, es una especie gregaria a lo largo del río donde forma los bosques llamados "capironales" (Spichiger et al., 1990; Brako \& Zarucchi, 1993). 
C. spruceanum tiene características ecológicas de pionera; la madera tiene densidad de $0.76 \mathrm{~g} / \mathrm{cm}^{3}$, color blanco pardo, muy dura, grano recto-entrecruzado, fácil aserrío y trabajabilidad, es utilizada en pisos, tarugos, molduras y estructuras; fue explotado en grandes volúmenes en la zona de Pucallpa (JUNAC, 1981; Aróstegui, 1982; Toledo, 1994).

\section{METODOLOGÍA}

En 1993, todos los individuos mayores de $8.5 \mathrm{~cm}$ de diámetro a la altura del pecho (DAP) fueron registrados en nueve parcelas permanentes de una hectárea y los individuos del sotobosque mayores de $1.5 \mathrm{~m}$ de altura hasta $8.5 \mathrm{~cm}$ de DAP fueron registrados en 24 subparcelas de $0.16 \mathrm{~m}$ x $0.16 \mathrm{~m}$, cubriendo un área total de 0.614 ha (Figura 1).

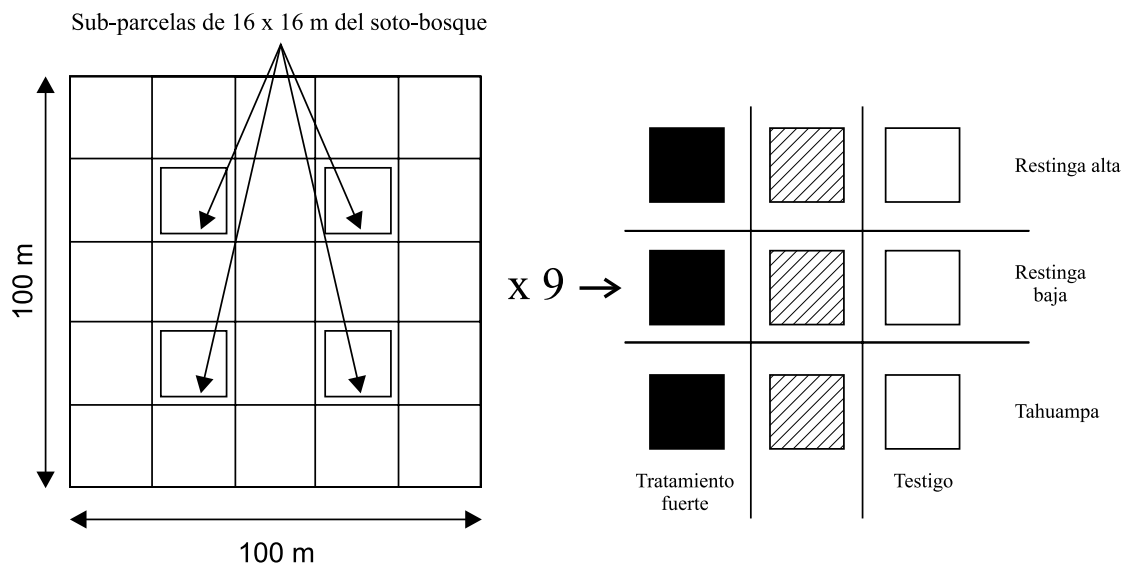

Figura 1. Diseño de parcelas permanentes de muestreo de 1 ha

Las nueve parcelas de una hectárea fueron distribuidos en tres parcelas en cada uno de los tres tipos de bosques: restinga alta, restinga baja y tahuampa; mientras que las sub parcelas del sotobosque fueron solamente establecidas en bosques de restinga. Después del establecimiento de las parcelas, a fines de 1993, se efectuaron tratamientos de talado de árboles a dos intensidades. En cada tipo de bosque una parcela fue testigo, mientras las dos parcelas adicionales fueron tratadas con apeo moderado ( $15 \%$ del área basal) y fuerte $(30 \%$ del área basal), respectivamente. Todos los 
individuos fueron remedidos en 1994, 1995, 1997 y 1999; los individuos de especies con madera de valor comercial fueron también remedidos en 1998. El ingreso de nuevos individuos fue registrado en 1995 y 1997. Los registros incluyeron medición de DAP, coordenadas y estimación de altura total (Nebel, 1999).

En 1996 y 1997, las parcelas permanentes de muestreo adicionales fueron establecidas en restinga alta con transectos de un ancho de $40 \mathrm{~m}$ y con un largo total de $2.4 \mathrm{~km}$ (9.6 ha, Figura 2). Todos los individuos mayores de $1.5 \mathrm{~m}$ de altura de especies seleccionadas con madera de valor comercial fueron registrados. Los registros incluyeron medición de DAP, coordenadas, proyección de copas y estimación de altura. El DAP de todos los individuos fue remedido en 1997, 1998 y 1999. Las plántulas de las especies seleccionadas fueron registradas y monitoreadas en 2400 parcelas de $2 \mathrm{~m} \times 2 \mathrm{~m}$, durante el período de tres años.

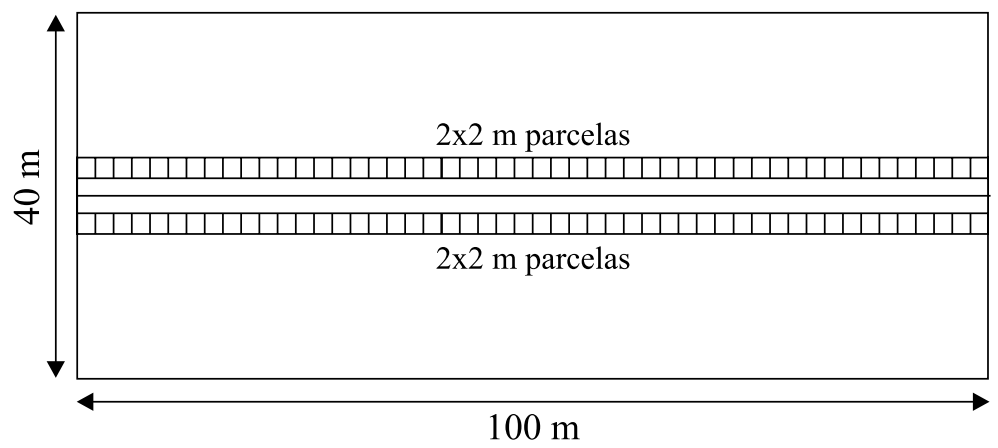

Figura 2. Parcela permanente de muestreo en forma de transecto 


\section{RESULTADOS Y DISCUSIONES}

\subsection{Estructura de la población y crecimiento de G. macrophylla}

Los individuos de G. macrophylla ocurren con una abundancia y una dominancia relativamente altas en los bosques de restinga alta, donde fueron registrados hasta 26 individuos por hectárea con más de $1.8 \mathrm{~m}^{2} / \mathrm{ha}$ (Tabla 1 ). La presencia de la especie en los otros dos tipos de bosques es considerablemente menor, aunque pequeños individuos fueron registrados, indicando que la especie podría estar en una fase de colonización de estos bosques, debido a una sucesión natural. Gracias a la abundancia relativamente alta de la especie se cuenta con una base de datos de un tamaño que hace posible diferentes análisis con resultados consistentes.

Tabla 1. Distribución de Guarea macrophylla por clases diamétricas en bosques inundables de la Amazonía peruana

\begin{tabular}{|c|c|c|c|c|c|c|c|c|}
\hline \multirow{2}{*}{$\begin{array}{l}\text { DAP } \\
(\mathrm{cm})\end{array}$} & \multicolumn{2}{|c|}{ Restinga alta } & \multicolumn{2}{|c|}{ Restinga baja } & \multicolumn{2}{|c|}{ Tahuampa } & \multicolumn{2}{|c|}{ Restinga alta } \\
\hline & $\begin{array}{c}\text { Abundancia } \\
\text { a } \\
(\mathrm{No} / \mathrm{ha})\end{array}$ & $\begin{array}{c}\text { Área } \\
\text { basal } \\
\left(\mathrm{m}^{2} / \mathrm{ha}\right)\end{array}$ & $\begin{array}{c}\text { Abundancia } \\
\text { a } \\
(\mathrm{No} / \mathrm{ha})\end{array}$ & $\begin{array}{c}\text { Área } \\
\text { basal } \\
\left(\mathrm{m}^{2} / \mathrm{ha}\right)\end{array}$ & $\begin{array}{c}\text { Abundancia } \\
\text { a } \\
(\mathrm{No} / \mathrm{ha})\end{array}$ & $\begin{array}{c}\text { Área } \\
\text { basal } \\
\left(\mathrm{m}^{2} / \mathrm{ha}\right)\end{array}$ & $\begin{array}{c}\text { Abundancia } \\
\text { a } \\
(\mathrm{No} / \mathrm{ha})\end{array}$ & $\begin{array}{c}\text { Área } \\
\text { basal } \\
\left(\mathrm{m}^{2} / \mathrm{ha}\right)\end{array}$ \\
\hline$<1$ & - & - & - & - & NA & NA & 0.1 & 0 \\
\hline $1-5$ & 9.8 & 57 & 6.5 & 0 & NA & NA & 6 & 0.0051 \\
\hline $5-10$ & 4 & 0.0033 & 3 & 0.0046 & NA & NA & 5.9 & 0.027 \\
\hline $10-20$ & 3.3 & 0.054 & 2.3 & 0.041 & 6 & 0.078 & 4 & 0.072 \\
\hline $20-30$ & 2.3 & 0.11 & - & - & - & - & 1.8 & 0.088 \\
\hline $30-50$ & 3 & 0.36 & 0.3 & 0.035 & - & - & 2.5 & 0.32 \\
\hline $50-70$ & 3.3 & 0.9 & - & - & - & - & 0.2 & 0.057 \\
\hline $70-$ & 7 & 0.4 & - & - & - & - & 0.2 & 0.11 \\
\hline Suma & 26.4 & 1.83 & 12.2 & 0.081 & 6 & 0.078 & 20.7 & 0.68 \\
\hline
\end{tabular}


Las alturas totales de la especie llegan a tamaños modestos, según el modelo de la relación entre DAP y altura total, basada en una función similar a la de Mitscherlich. El resultado ajustado en el paquete SAS utilizando el procesamiento de NLIN es: (Figura 3)

$$
h=30.75 \cdot\left(1-0.9429 \cdot e^{-0.03079 \cdot d}\right)
$$

Donde $h$ es la altura (m) del árbol y $d$ es el DAP (cm).

El incremento diamétrico promedio anual de todos los individuos de G. macrophylla subió con los tamaños de las clases diamétricas, mostrando el valor más alto en la clase diamétrica de árboles más grandes de $70 \mathrm{~cm}$ DAP (Figura 3). Considerando solamente el promedio de los cinco máximos incrementos diamétricos de cada clase diamétrica, se observa que existe una potencialidad grande de incremento en la especie, tomando en cuenta que incrementos diamétricos hasta $30 \mathrm{~mm} /$ año fueron observados. El tiempo que necesita un árbol para llegar al tamaño de $90 \mathrm{~cm}$ DAP es 164 y 57 años aplicando las tasas de incremento diamétrico promedio y de los cinco árboles con incremento máximo de cada clase diamétrica, respectivamente (Figura 3). 

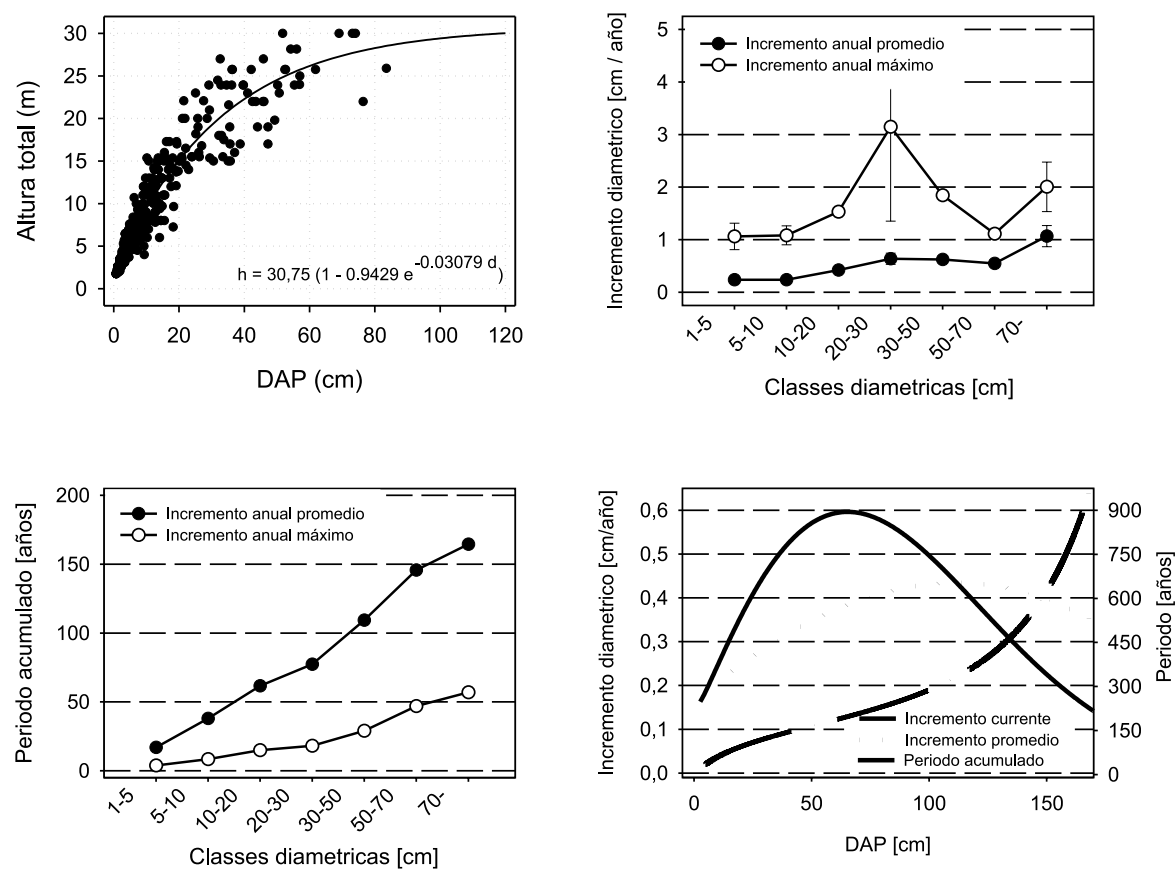

Figura 3. Guarea macrophylla: (a) observaciones y modelo de la relación entre DAP y la altura total, (b) incremento diamétrico por clases diamétricas promedio de todos los individuos y promedio de los cinco individuos con máximo incremento, (c) período acumulado para que un árbol llegue al tamaño de un clase diamétrica - suponiendo el incremento promedio de todos individuos y el promedio de los cinco individuos con máximo incremento, (d) incremento corriente anual, incremento promedio anual y tiempo acumulado para llegar a un cierto diámetro según un modelo de crecimiento.

Un modelo de incremento diamétrico fue ajustado en el paquete de SAS a una función donde DAP es la única variable (Figura 2), con un resultado aceptable $\left(\mathrm{R}^{2}=0.21\right.$ y $\left.\mathrm{F}<0.0001\right)$ : 


$$
\Delta d=\sqrt{e^{-6.5656+1.5016 \cdot \ln (d)-0.000175 \cdot d^{2}+7.5337 \cdot e^{-\sqrt{d}}}}
$$

Donde $\Delta d$ es el incremento anual de DAP (cm) y $d$ es el DAP (cm).

Según el modelo (2) el pico del incremento corriente anual llega a $6 \mathrm{~mm}$ /año y ocurre a un diámetro alrededor de $60 \mathrm{~cm}$ DAP, mientras el incremento promedio anual tiene su pico con un tamaño alrededor de $110 \mathrm{~cm}$ DAP donde llega a un valor de $4 \mathrm{~mm} / \mathrm{año}$ (Figura 3).

Los resultados del modelo de crecimiento muestran un incremento de diámetro más lento de los resultados calculados como promedio de las clases diamétricas, y considerablemente más lento de los incrementos máximos observados en la especie (Figuras 2 y 3). Sin embargo, los resultados pueden dar una idea del nivel de incremento que tienen los árboles de esta especie. En el futuro es recomendable incluir factores de competencia en los análisis de incremento para identificar y cuantificar los factores que causan las diferencias en el incremento en diámetro observados en los datos.

\subsection{Estructura de la población y crecimiento de $C$. spruceanum}

Una limitación relevante con C. spruceanum en este trabajo es la baja abundancia de la especie en los bosques donde fueron establecidas las parcelas permanentes de muestreo, resultando una base de datos con pocas observaciones. Esto se debe a que la especie ecológicamente tiene características de pionera, mientras las parcelas en su mayoría fueron establecidas en lugares con bosques maduros con claros de pequeños tamaños. Por lo tanto, se encontró pocos individuos de la especie y mayormente de tamaños grandes (ver observaciones de altura en el Figura 4), implicando que los resultados del presente trabajo sean muy débiles y no correspondan bien al conocimiento de la especie como pionera y con rápido incremento diamétrico.

La relación entre DAP y altura total, basada en una función similar a la de Mitscherlich, muestra que la especie llega relativamente a grandes alturas totales. El resultado de la función, ajustado en el paquete de SAS, utilizando el procesamiento de NLIN es: (Figura 4)

$$
h=42.22 \cdot\left(1-0.9763 \cdot e^{-0.0232 \cdot d}\right)
$$

Donde $h$ es la altura (m) del árbol y $d$ es el DAP (cm). 
No fue posible analizar los incrementos de diámetro en clases diamétricas de pequeños tamaños, debido a la falta de observaciones. Recién en clases diamétricas a partir de $10 \mathrm{~cm}$ de DAP se tiene algunas observaciones, pero, con niveles de crecimiento muy bajos que no guarda correspondencia con la característica de la especie que cuenta con rápido crecimiento desde su establecimiento en el bosque. En clases diamétricas más grandes se puede observar incrementos más rápidos (Figura 4). Debido a la carencia de datos en clases diamétricas pequeñas e incrementos relativamente lentos, demora mucho tiempo para llegar a ciertos tamaños.

Con base a datos disponibles de las parcelas permanentes el incremento diamétrico fue modelado en el paquete SAS, utilizando una función donde DAP es la única variable (Figura 4$)$, con un resultado no muy bueno $\left(\mathrm{R}^{2}=0.18\right.$ y $\left.\mathrm{F}<0.01\right)$ :

$$
\Delta d=\sqrt{e^{-13.3655+3.0895 \cdot \ln (d)-0.000177 \cdot d^{2}+24.14 \cdot e^{-\sqrt{d}}}}
$$

Donde $\Delta d$ es el incremento anual de DAP (cm) y $d$ es el DAP $(\mathrm{cm})$.

El modelo muestra un incremento de diámetro relativamente lento que probablemente se debe a la falta de datos y con bajos niveles de incremento. 

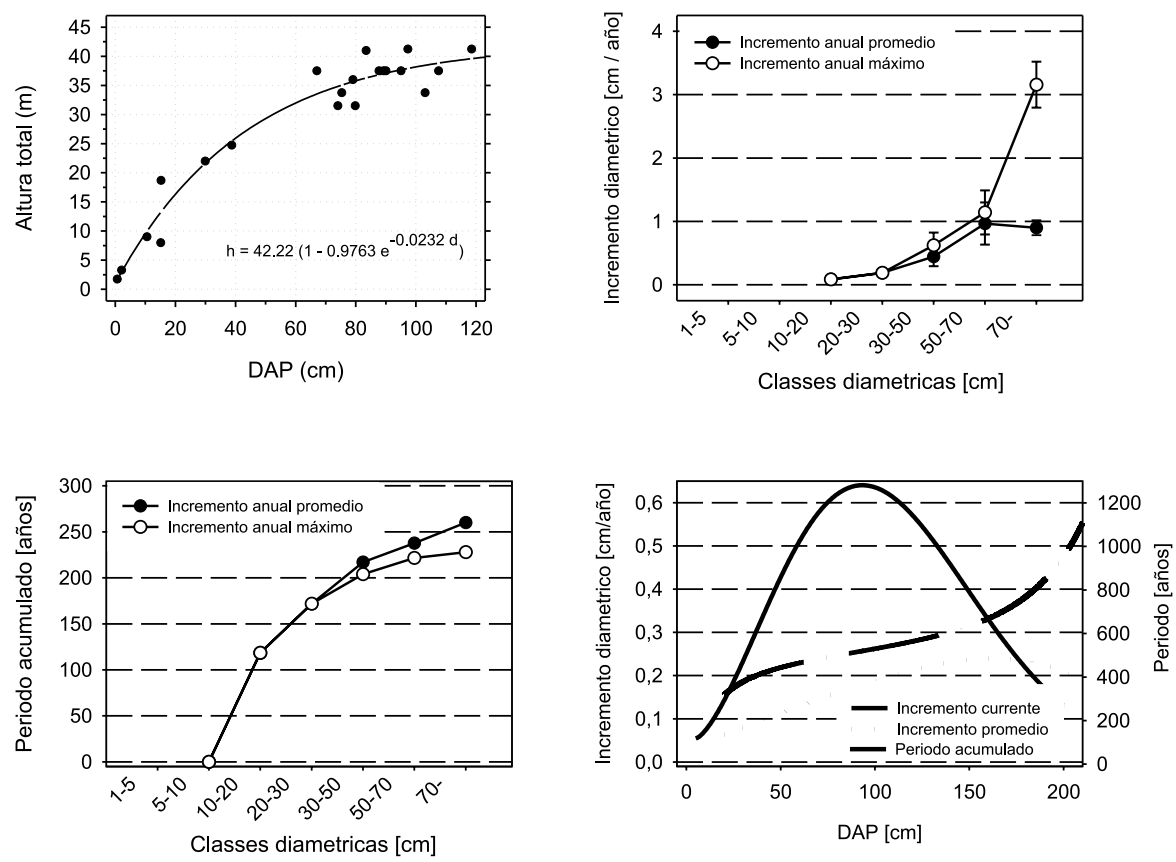

Figura 4. Calycophyllum spruceanum: (a) observaciones y modelo de la relación entre DAP y la altura total, (b) incremento diamétrico por clases diamétricas - promedio de todos los individuos y promedio de los cinco individuos con máximo incremento, (c) periodo acumulado para que un árbol llegue al tamaño de una clase diamétrica - suponiendo el incremento promedio de todos individuos y el promedio de los cinco individuos con máximo incremento, (d) incremento corriente anual, incremento promedio anual y tiempo acumulado para llegar a un cierto diámetro según un modelo de crecimiento. 


\section{BIBLIOGRAFÍA}

ARÓSTEGUI, A. 1975. Estudio tecnológico de maderas del Perú (zona Pucallpa) Vol I; características tecnológicas y usos de la madera de 145 especies del país. Ministerio de Agricultura, Universidad Nacional Agraria - La Molina, Lima - Perú. 483 p.

BRAKO, L. \& ZARUCCHI, J. 1993. Catálogo de las Angiospermas y Gimnospermas del Perú. Missouri Botanical Garden. Segunda reimpresión 1996. 1286 p.

JUNAC. 1981. Tablas de propiedades físicas y mecánicas de la madera de 20 especies del Perú. PADT REFORT. Lima, Perú. 53 p.

LAO, R. 1986. Descripción dendrológica de 51 especies forestales Asentamiento Rural Forestal von Humboldt. Huánuco, Perú.

NEBEL, G. 1999. Ecology and management of flood plain forests in the Peruvian Amazon. Ph.D. dissertation, The Royal Veterinary and Agricultural University, Copenhagen, Denmark. 179 p.

SPICHIGER, R.; MEROZ, J.; LOIZEAU, P.A. \& STUTZ DE ORTEGA, L. 1990. Contribución a la flora de la Amazonía peruana; los árboles del arborétum Jenaro Herrera. V 2. Conservatorio y Jardín Botánicos de la ciudad de Ginebra, Organización Suiza para el Desarrollo y la Cooperación, Instituto de Investigaciones de la Amazonía Peruana. Ginebra, Suiza. 565 p.

TOLEDO, E. 1994. Mercado y desarrollo de la industria forestal con especies de maderas no tradicionales. Documento presentado en el Seminario Taller "Biodiversidad y desarrollo sostenible en la Amazonía en una economía de mercado". Pucallpa 11-15 Octubre, Perú. 46 p. 